\title{
Modelling Upwelling Irradiance using Secchi disk depth in lake ecosystems
}

\author{
Luca BRACCHINI, Arduino M. DATTILO, Vincent HULL ${ }^{1)}$, Steven Arthur LOISELLE, \\ Antonio TOGNAZZI and Claudio ROSSI \\ Environmental Spectroscopy Group, Dipartimento di Scienze e tecnologie Chimiche e dei Biosistemi, Via A. Moro 2,53100 Siena \\ and Consorzio Interuniversitario per lo sviluppo dei sistemi a grande interfase, CSGI, Via della Lastruccia 2, Firenze \\ ${ }^{1)}$ Consiglio per la ricerca e la sperimentazione in agricoltura, Via Nazionale 82, 00183, Roma, Italia \\ *e-mail corrsponding author: bracchini@unisi.it
}

\begin{abstract}
A simple model for upwelling irradiance has been developed. The model represents the relationship between Photosynthetically Active Radiation diffuse attenuation coefficients and Secchi disk depth described with a physical-mathematical expression. This physical mathematical expression allows the evaluation of the sub surface upwelling irradiance that was generated by the interaction between downwelling irradiance and the water column. The validation of the relation was performed using experimental data collected from five different aquatic ecosystems at different latitudes, solar elevations and irradiance levels. We found a good linear, positive correlation between the theoretical and measured upwelling irradiance $\left(\mathrm{R}^{2}=0.96\right)$. The residues were well distributed, around the null value, according a Gaussian curve $\left(\mathrm{R}^{2}=0.92\right)$. The results confirm the importance and the versatility of the Secchi disk measurements for aquatic optics.
\end{abstract}

Key words: limnology, PAR attenuation coefficient, solar irradiance, lakes

\section{INTRODUCTION}

The oldest, simplest, most inexpensive and used water optical instrument is the Secchi disk, a $20 \mathrm{~cm}$ diameter white disc lowered in the water till its disappear by human eye. The depth (m) in which the Secchi disk is not visible is the measured Secchi disk depth. The first recorded measurement of the Secchi disk depth took place in 1865 and was performed by its inventor, Abbot Angelo Secchi (1818-1878, Tricomi 1962) during the first Mediterranean physical research cruise, funded by the Papal State. Despite the simplicity of the instrument, the measurement procedure and its interpretations are not trivial. In order to interpret the Secchi disk measurements, Preisendorfer (1986) published the "Ten Laws for the Secchi Disk", but still a number of different approaches in the use of this simple tool but effective persist. To give an example, oceanographers made use of different versions of the Secchi disk: all white disks, or black and white quadrant disks with different diameters. Moreover, Verschuur (1997) define the Secchi disk depth as the depth at which the disk was "just visible", Effler (1988), as the depth at which the disk disappears, and others as the average value between the two above (Davies-Culley et al. 1993; Wetzel \& Likens 1991).

The Secchi disk depth, as a physical measure, is an Apparent Optical Property (AOP) of the medium, such as the measured irradiance ( $\mathrm{W} \mathrm{m}^{-2}$, or spectral irradiance, $\mathrm{W} \mathrm{m}^{-2} \mathrm{~nm}^{-1}$ ), the downward/upward diffuse attenuation coefficients $\left(\mathrm{m}^{-1}\right)$ and the irradiance reflectance (dimensionless). AOP are optical properties that describe the behaviour of water bodies in a particular light field (Preisendorfer 1961). On the contrary, the Inherent Optical Properties (IOP) are the optical properties of the medium independent of the light field (Mobley 1994); absorption coefficients $\left(\mathrm{m}^{-1}\right)$, volume scattering function $\left(\mathrm{sr}^{-1} \mathrm{~m}^{-1}\right)$, and scattering/backscattering coefficients $\left(\mathrm{m}^{-1}\right)$ are examples of IOP. A wide debate between authors (Gordon \& Wouters 1978; Preisendorfer 1986; Kirk 1984; Haltrin et al. 2002) arose in order to decide whether the AOP Secchi disk depth could be used as an indirect measure of some IOP and, more in general, whether it is possible to obtain some relationship between IOP and AOP.

Relations between the Secchi disk depth and other optical properties were investigated by Tyler (1968). He applied the contrast transmittance theory to explain the measured depth of the Secchi disk. Secchi disk depth $\left(Z_{d}\right)$ is inversely proportional to the sum of the beam attenuation coefficients $(c=a+b$, where $\mathrm{a}$ is the absorption and $b$ is the scattering) and diffuse attenuation coefficient $\left(K_{d}\right)$ obtaining $c+K_{d}=8.69 \times Z_{d}^{-1}$. Using the same relationship, Holmes (1970) found a value of 9.42. In some cases, $c$ and $K_{d}$ covary thus the inverse relationship can be also applied neglecting $c$ (Kirk 1994). An inverse relation can be written to express the dependency between the Secchi disk depth and the organic and inorganic matter in the water (Jassby et al. 1999). Poole \& Atkins (1929) found an inverse relation between the Secchi disk depth and the diffuse attenuation coefficient in visible band. More recently, the same relationship was found using a quanta irradiance meter (Hojerslev \& Aarup 2002). Padial \& Thomaz (2008) found, analysing a large data set, a new 
value for the constant that links the inverse relationship between Photosynthetically Active Radiation diffuse attenuation coefficient $\left(K_{d, P A R}\right)$ with the Secchi disk depth. Therefore, the importance of the Secchi disk measure is related also to the possibility to obtain an estimation of the $K_{d, P A R}$, or in the visible band, without using an expensive quanta-meter or spectroradiometer.

In this work, we propose a simple model to write a physical-mathematical expression for the constant that links the Secchi disk depth with the $K_{d, P A R}$, which allows an estimate of the upwelling irradiance, just below the water surface.

\section{METHODS}

In order to study the upwelling irradiance in aquatic environments the global irradiance (composed by direct and diffuse irradiance beams), applied to a stratified medium, can be used to study the water radiative transfer (Sokoletsky 2005). We analyze the homogeneous single layer medium for the PAR irradiance, then define $I_{0, P A R}$ as the PAR band irradiance just below the surface water; $r_{P A R}$ as the reflectance of the bottom of the layer; and $\Delta \mathrm{h}$ as the thickness of the layer. If the attenuation coefficients for downward and upward irradiance are the same (called here $k_{P A R}$ ), we can write the upwelling irradiance in the PAR band ( $E_{u w . P A R}$ equation 1$)$ as follows:

$$
E_{u w, P A R}=E_{0, P A R} \cdot r_{P A R} \cdot e^{-\frac{2 k_{P A R} \Delta h_{1}}{\cos \theta}}
$$

where $\vartheta$ is the angle between $E_{0, P A R}$ and the normal to the surface, and to take account of the total path length, we multiply the exponent by a factor of 2 .

The global irradiance and the diffuse attenuation coefficient for downward PAR irradiance were represented by:

$$
E_{P A R}(z)=E_{0, P A R} e^{-K_{d, P A R}\left(z-z_{0}\right)}
$$

where $E_{0, P A R}$ is the PAR irradiance at depth $z_{0}$ (in our case $\left.z_{0}=0.02 \mathrm{~m}\right), E_{P A R}(z)$ is the irradiance measured at depth $z(\mathrm{~m})$, and $K_{d, P A R}\left(\mathrm{~m}^{-1}\right)$ is the diffuse attenuation coefficient for the downwelling PAR irradiance. The $K_{d, P A R}$ were calculated using the vertical depth $\left(z-z_{0}\right)$; it should be noted that in equation 1 attenuation coefficients $\left(k_{P A R}\right)$ is referred to the path length of the direct irradiance. Then the geometrical relation between $k_{P A R}$ and $K_{d, P A R}$ is:

$$
K_{d, P A R} \cos \vartheta=k_{P A R}
$$

Therefore, substituting equation (3) in equation (1) we obtain:

$$
E_{u w, P A R}=E_{0, P A R} \cdot r_{P A R} \cdot e^{-2 K_{d, P A R} \Delta h}
$$

If we consider as $A_{d}$ the measured Secchi disk depth, in according to Verschuur (1997) we correct $A_{d}$ as:

$$
\begin{aligned}
& Z_{d}=\frac{A_{d} \cdot(1+F)}{2 F} \text { with } \\
& F=\cos \left(\arcsin \left(\frac{\sin \phi}{1.33}\right)\right)
\end{aligned}
$$

where 1.33 is the refraction index between air and water and $\phi$ the angular inclination of sun to the zenith and $Z_{d}$ (m) is the corrected Secchi disk depth.

An empirical relation between Secchi disk depth $\left(Z_{d}\right)$ and the $K_{d, P A R}$ is well represented by an hyperbolic function (equation 5, Poole \& Atkins (1929), Tyler (1968), Hojerslev \& Aarup (2002), Padial \& Thomas (2008)).

$$
Z_{d} \cdot K_{d, P A R}=C O N S T
$$

If the medium is not stratified or the Secchi disk depth is less than the depth of the boundary of the first layer, and if the $\Delta h$ with $Z_{d}$ and $r_{P A R}$ is substitute with the irradiance reflectance of Secchi disk $\left(r_{P A R}\right.$, dimensionless), then we can rewrite, for a single layer, the equation 4 as:

$$
E^{S}{ }_{u w, P A R}=E_{0, P A R} \cdot r_{P A R} \cdot e^{-2 \cdot K_{d, P A R} \cdot Z_{d}}
$$

where $E_{u w, P A R}^{S}$ is the upwelling irradiance generated by the Secchi disk reflection.

Equation 7 represent the portion of upwelling irradiance that is generated from the reflection on the Secchi disk when $E_{0, P A R}$ attenuation is described by $K_{d, P A R}$. Even if $K_{d, P A R}$ describe the absorption and the scattering of the irradiance, $E_{u w, P A R}^{S}$ generated by the reflection of the Secchi disk may be, in general, less than the measured upwelling irradiance at the sub surface depth. In fact, the latter is generated by the back scattering of each suspended and dissolved particles that are present in the medium from sub surface depth of the water column to the bottom of the lake (especially if the lake is shallow). A possible solution to this discrepancy is adopted considering, instead to the actual Secchi disk reflectance an "apparent reflectance" $\left(\widetilde{r}_{P A R}\right)$, that which considers the contribution at upwelling irradiance of water column between sub surface depth and Secchi disk depth. Then a modified equation 7 can be written as (equation 8 ):

$$
E_{u w, P A R}=E_{0, P A R} \cdot \widetilde{r}_{P A R} \cdot e^{-2 \cdot K_{d, P A R} \cdot Z_{d}}
$$

with $E_{u w, P A R}$ the upwelling irradiance of the whole water column between sub surface and the Secchi disk depth. In other words, $\widetilde{r}_{P A R}$ can be also considered as the new Secchi disk irradiance reflectance as if all particles that are between the Secchi disk and sub surface depth were just above the Secchi disk. This assumption take account also that the upwelling irradiance depend also by the quality and quantity of the optically active component in the water column in a specific site as well as the variation of the actual Secchi disk reflectance with years. 


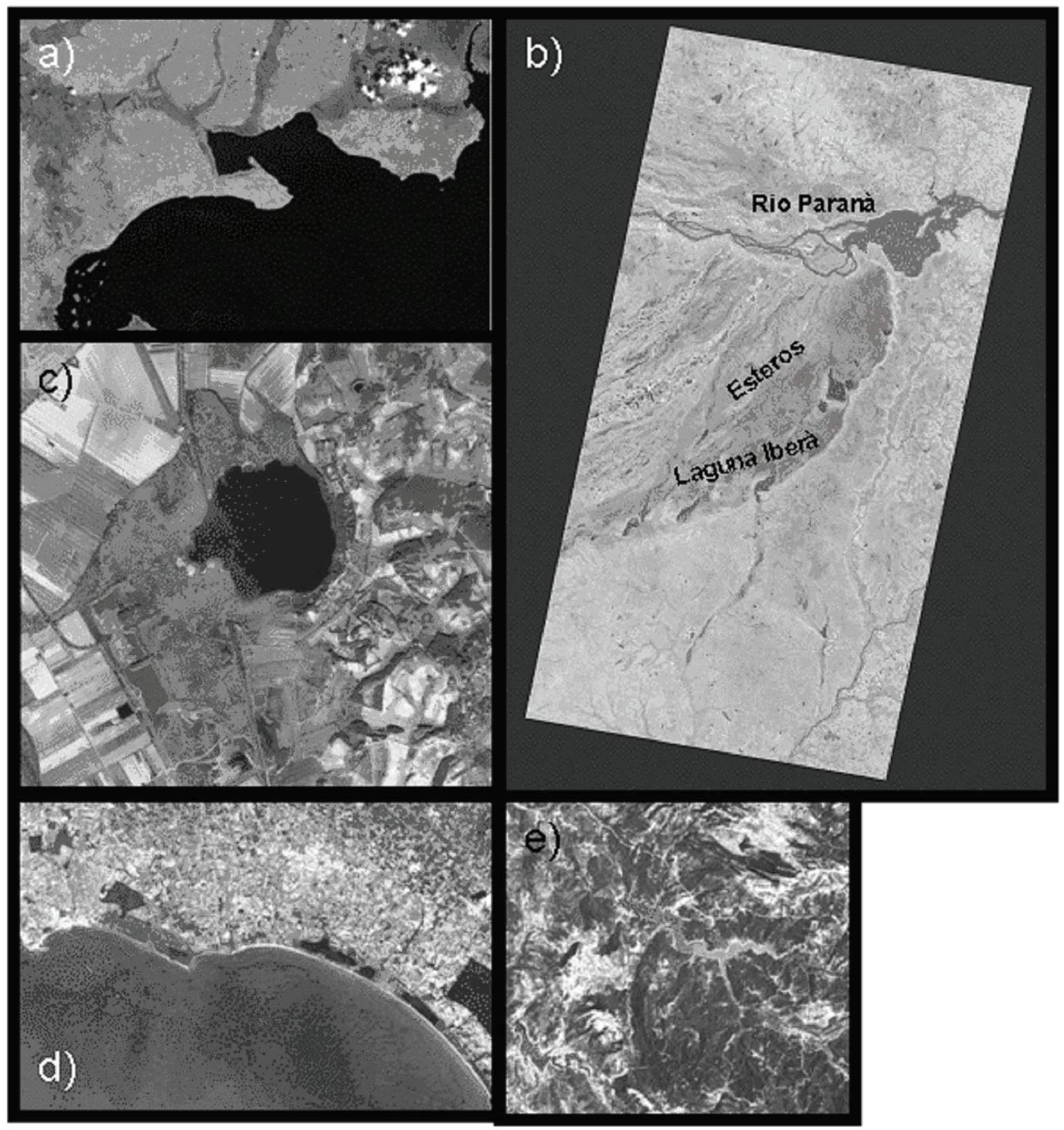

Fig. 1. a) Katonga and Bunjako Bays (Lake Victoria, Uganda); b) Laguna Iberà (Esteros del Iberà, Argentina); c) Lake of Montepulciano (Montepulciano Reserve, Province of Siena, Italy); d) Lake of Fogliano (Circeo National Park, Province of Latina, Italy); Lake of Salto (Province of Rieti, Italy)

From equations 6 and 8 it is possible to explicit the mathematical-physical interpretation of CONST (equation 9):

$$
E_{u w, P A R}=E_{0, P A R} \cdot \widetilde{r}_{P A R} \cdot e^{-2 \cdot C O N S T}
$$

Thus:

$$
Z_{d} \cdot K_{d, P A R}=C O N S T=-\frac{1}{2} \operatorname{Ln}\left(\frac{E_{u w, P A R}}{E_{0, P A R}} \cdot \frac{1}{\widetilde{r}_{P A R}}\right)
$$

\section{MATERIAL}

To validate the proposed model all measurements were collected in lake ecosystems. The data were collected in Katonga and Bunjako Bays, Laguna Iberà, Lake of Montepulciano, Lake of Fogliano and Lake of
Salto (Fig. 1). Katonga and Bunjako Bays (UTMx 392 Km, UTMy 9994 km, Victoria Lake, Uganda, figure 1a) are located in the Ugandan section of Lake Victoria. These bays are located at an equatorial latitude. The former is surrounded by papyrus dominated littoral wetlands on three sides and has a surface area of 10 $\mathrm{km}^{2}$. Katonga Bay receives inflow from the Katonga River in its north-western corner. Bunjako Bay $\left(30 \mathrm{~km}^{2}\right)$ is located between Katonga Bay and the open Lake Victoria area. This bay has a lower percentage of coastal wetland $(15 \%)$. Both bays are characterized by a maximum depth of $10 \mathrm{~m}$. In this ecosystem was measured high concentration of chromophoric dissolved organic matter (CDOM) released by wetland ecosystem. Consequently, the penetration of the solar UV and PAR irradi- 
ance is limited in particular near the coast line (Bracchini et al. 2006a; Bracchini et al. 2007). A single survey was conducted in this lake and data were acquired in 46 stations. Laguna Iberà (UTMx $484 \mathrm{~km}$, UTMy $6848 \mathrm{~km}$, Province of Corrientes, Argentina, figure 1b) is a Ramsar wetland of international importance and is characterised by a significant biodiversity. The lake (open water area $54 \mathrm{~km}^{2}$ ) is a permanent lake with an average depth of $3 \mathrm{~m}$ and a maximum of $4.5 \mathrm{~m}$. The lake has two small rivers which drain wetland areas into the southern and northern parts of the lake. The lake borders consist of floating vegetation mats. These mats extend up to $1 \mathrm{~km}$ in width and 2 meters in depth and consist of living vegetation in a substrate of degraded plant matter which is in contact with water below and on each side. Dissolved organic matter and humic substances are constantly being released by the vegetation mats into the surrounding water. Also this lake is characterized by high CDOM absorption and high spatial variation of inherent and apparent optical properties were observed (Bracchini et al. 2004). The human activities around the lake are low. A single survey was conducted in this lake and data were acquired in 77 stations. Lake of Montepulciano (UTMx $738 \mathrm{~km}$, UTMy $4775 \mathrm{~km}$, Province of Siena, Italy; figure 1c) is a small size lake $\left(\sim 1 \mathrm{~km}^{2}\right)$ in the southern part of Province of Siena. The maximum depth of this lake was $3 \mathrm{~m}$. The lake is a part of "Montepulciano Reserve" that it is one of the most important Italian sites for the birds migration and reproduction. The coastal line of the lake is dominated by the presence of vegetation and the agricultural activities is high. Thus, high concentration of nutrients and eutrophic condition were observed. The lake is characterized by high turbidity due to the presence of high concentration of phytoplankton. Four surveys were conducted in this lake and data were acquired in 10 station each one. Lake of Fogliano (UTMx 324 $\mathrm{km}$, UTMy $4586 \mathrm{~km}$, Province of Latina, Italy, figure 1d) is a lake of the Pontine Lagoons located $100 \mathrm{~km}$ south of the city of Rome (Italy), in the territory of the National Park of Circeo. The Lake of Fogliano (surface $4 \mathrm{~km}^{2}$ ) is the largest of the three park lakes. The south west part of the lake is separated from the Tyrrhenian Sea by a coastal sandy dune characterized by typical Mediterranean coastal vegetation. The north-easternmost territory, off the lake borders, is characterized by an extensive agriculture area with zootechnical activities, mainly water buffalo. A limited exchange, between the marine and lake waters, occurs through a $10 \mathrm{~m}$ wide canal, with the flowrate being controlled by tides. All freshwater inputs have been deviated to reduce pollutant entrainment into the lake, this has led to a strong increase in salinity (average value above $40 \mathrm{~g} \mathrm{~L}^{-1}$ ), in the last two decades. The average depth of the lake is around $1 \mathrm{~m}$. The bottom is characterized by the presence of submerged macrophytes (Ruppia maritima and Cymodocea nodosa), and a sediment consisting mainly of sandy silt. Wind direction and velocity is measured automatically at the lake border and shows typical sea breeze regimes. The wind resuspention greatly influencing the optical properties of the lake (Bracchini et al. 2005). Thus, we have used data (40 values of PAR diffuse attenuation coefficients and Secchi disk depths) from surveys in which the wind velocity was low (less than $2.2 \mathrm{~m} \mathrm{~s}^{-1}$ ). Lake of Salto (UTMx $341 \mathrm{~km}$, UTMy $4679 \mathrm{~km}$, Province of Rieti, Italy, figure 1e) is an artificial lake created by a dam located in the north-west. The most important river inlet, in the south-eastern part of the lake, receives snow melt from the surrounding mountains, while some smaller rivers are present in the spring season. The outflow from the lake is controlled for hydroelectric production and the lake exhibits daily and seasonal variations in the water level up to $10 \mathrm{~m}$. The lake is characterized by a minimum depth of $3 \mathrm{~m}$ near the river inlet and maximum depth about of $60 \mathrm{~m}$ near the dam. Littoral areas are composed of clay and sand, and vegetation is limited. Aquatic macrophytes are present near the river inlet and along the coast. Small population centres and agricultural activities are present in the northern catchment. This is the most clear of the considered lakes but the CDOM photodegradation greatly influencing the radiative transfer in the UV band during the summer season. On the contrary, the $K_{d, P A R}$ do not change significantly during the year (Bracchini et al. 2006b). Data from a summer survey was used visiting 26 stations in the lake. From each lake, 14 additional sampling stations were used to validate the proposed model with exception of the Lake of Salto in which 15 sampling stations were used.

In order to measure the $E_{d, P A R}\left(\mu \mathrm{E} \mathrm{m}^{-2} \mathrm{~s}^{-1}\right)$ profiles, a Teflon-covered quartz cosine response PAR sensor mounted on a PUV 541 spectroradiometer (SN 19235, Biospherical Instruments, San Diego, CA) was used. The relative error by cosine response of the sensor is less than $\pm 2 \%$ from $0^{\circ}$ to $65^{\circ}$ (angle between the normal of the sensor and the irradiance vector) and less than $\pm 10 \%$ from $65^{\circ}$ to $85^{\circ}$. During the measurements, the PUV541 was connected to a portable battery and a portable computer to collect irradiance data. The instrument was lowered into the water column and measurements of $E_{d, P A R}$ and water depth were acquired simultaneously every 0.3 seconds. The PAR irradiance measurements were initiated below the water line $(0.02$ $\mathrm{m})$ and were recorded while the instrument was lowered until close to the bottom, same measures were collected raising the instrument just below the water surface. On average, between 100-1000 measurements (PAR irradiances and water depth $(\mathrm{m})$ ) were obtained at each profile. The resulting irradiance profile was corrected by removing the dark current signal and plotted against the water depth. The PUV was lowered in the sun exposed side of the boat.

The upwelling PAR irradiance $\left(E_{u w, P A R}\right)$ was measured at $0.02 \mathrm{~m}$ under the water surface with the PUV 


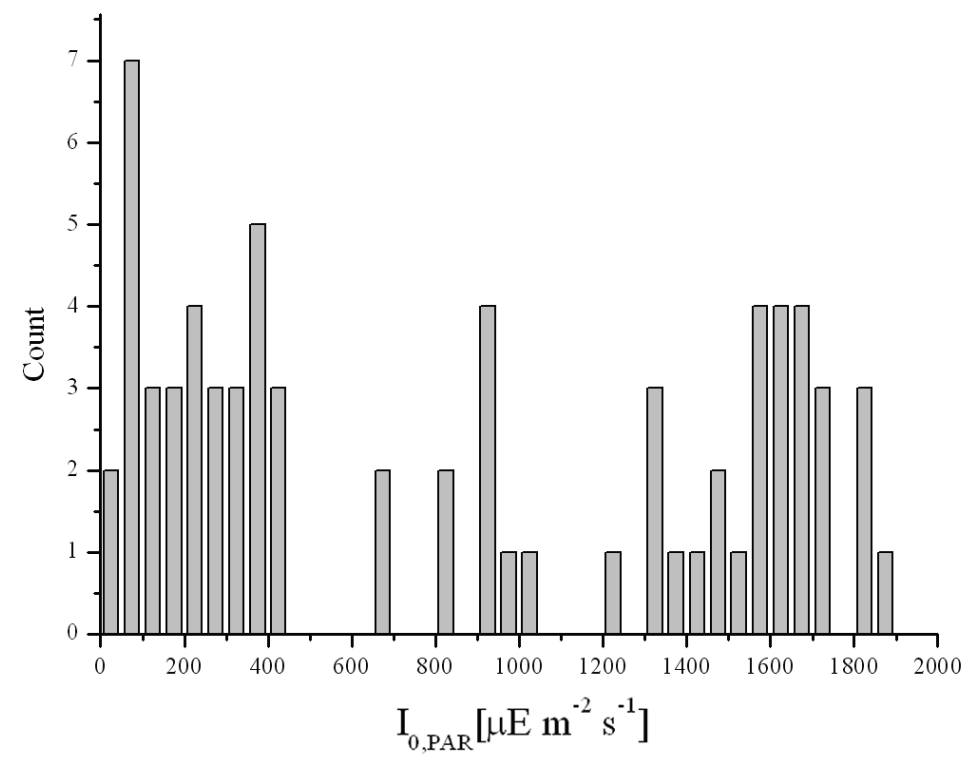

Fig. 2. Measured PAR irradiance just below the water surface used in the validation of the model for the upwelling irradiance (71 measurements). Different insulation condition could be noted. The measurements were collected from 5 different aquatic ecosystems.

instrument in 71 different sampling points in extreme different insulation conditions (Fig. 2). In the 71 sampling points PAR irradiance profile were collected, but only the $E_{0, P A R}$ and $E_{u w, P A R}$ irradiances values were used to validate the proposed model.

In correspondence of PAR irradiance profiles, 229 values of Secchi disk depth $\left(A_{d}\right)$ were measured. The used Secchi disk (the same Secchi disk in all surveys) was characterized by a planar white circular panel of $0.20 \mathrm{~m}$ of diameter attached to a graduate line with precision of $0.02 \mathrm{~m}$. The depth of Secchi disk was determined with average values between the depth where the disk was distinguishable in the aquatic medium and the depth at which the disk disappeared at human eye. Thus, the uncertain of the Secchi disk depth measurements may be related to the specific person that perform the measure. The same four different people were involved in the measurements in each lake, thus, we believe that differences in the sensitivity of the human eye should be mediated during the sampling plane in each lake. Moreover, at the beginning of our measurements (year 2001) we try to change the people involved in the Secchi disk depth measurements and the values were the same (differences less than $0.06 \mathrm{~m}$ ) for low depths and to not exceed $0.20 \mathrm{~m}$ for Secchi disk depth greater than 2.00 $\mathrm{m}$. The corrected Secchi disk depth $\left(Z_{d}\right)$ was calculated according to the equation (5).

The measure conditions were characterized by low speed wind and by the absence of water waves that could influence the measurements (Hojerslev \& Aarup 2002). The collection of the measurements were made between $10.00 \mathrm{am}$ and $15.00 \mathrm{pm}$ local hour. The time of measurements and the geographic positions of every sampling point were collected using a portable GPS (Garmin).
PAR diffuse attenuation coefficients were estimated using a non linear least square fitting and 229 values of $K_{d, P A R}$ are determined according to the equation (2). For each fitted profile the residuals between the theoretical exponential curve and the measured PAR downwelling irradiance was checked to be distributed around the null value. In addition, the absence of optical stratification in the PAR band was also checked.

In order to measure the reflectance of the Secchi disk we used a StellarNet2000 Spectroradiometer (StellarNet Inc., USA), equipped with two standard lamps (LS1 in the visible wavelengths and LS3, in the UV wavelengths, StellarNet Inc., USA) light, to obtain a reference and fibre optics. The reference was used to illuminate the disk using a fibre optic. The fibre was deployed normally to the surface of the Secchi disk. A second fibre optic was used to collect the reflected light. Also in this case the intensity was collected in a normal configuration. The Secchi disk reflectance was obtained as the ratio between the intensities of reflected and the reference lights.

\section{RESULTS}

The value of CONST was found using equation 6 , data of $Z_{d}(10)$, and $K_{d, P A R}(2)$ (Fig. 3).

$C O N S T \pm \triangle C O N S T=1.80 \pm 0.02$ [dimensionless] with $N=229$ and $R^{2}=0.81$ have been computed. The distribution of the residuals between the theoretical hyperbolic function and experimental data can be described using a Gaussian distribution. Considering $0.25 \mathrm{~m}^{-1}$ the step size of the classes, the expected value of the best Gaussian curve fitting is $-0.09 \pm 0.02 \mathrm{~m}^{-1}$ and the standard deviation is $0.35 \pm 0.04 \mathrm{~m}^{-1}$ (Fig. 4).

It is possible to obtain the theoretical upwelling PAR irradiance $\left(E_{u w, P A R}\right)$ by substituting the value of CONST 


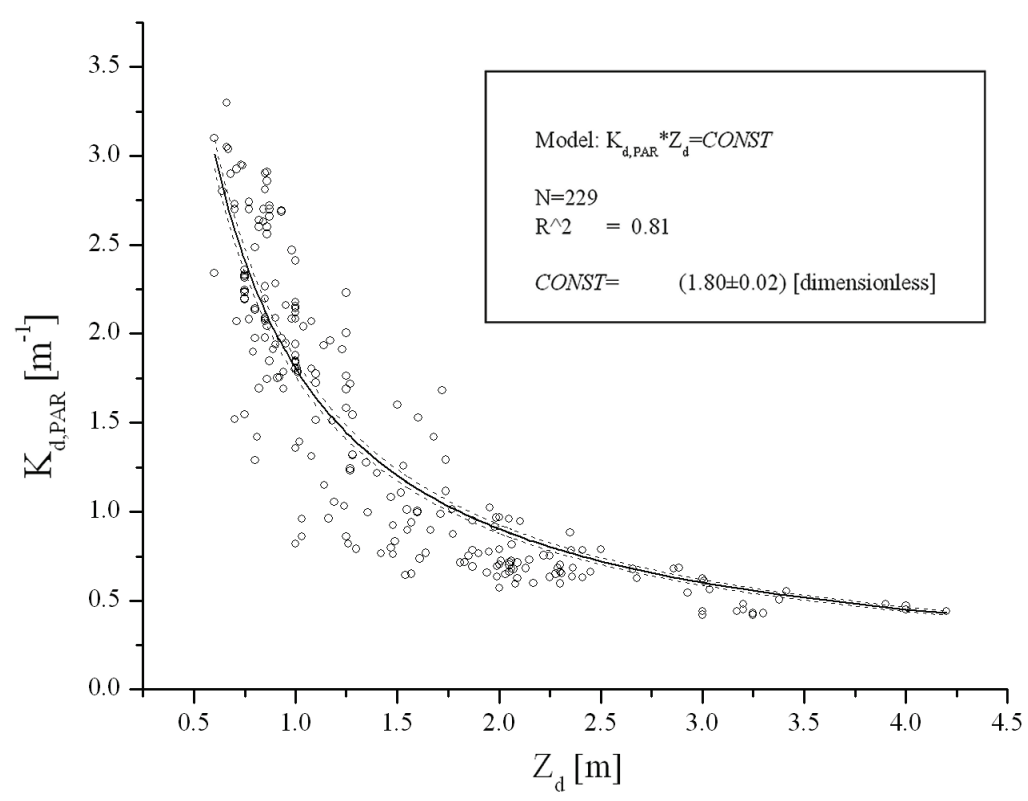

Fig. 3. Theoretical and measured hyperbolic relation between the Secchi disc depth and PAR diffuse attenuation coefficients. With the 229 couple of values it is possible determining CONST $\pm \triangle C O N S T=1.80 \pm 0.02$. Solid line represent the theoretical hyperbolic function. Dash lines represents the $95 \%$ band confidence.

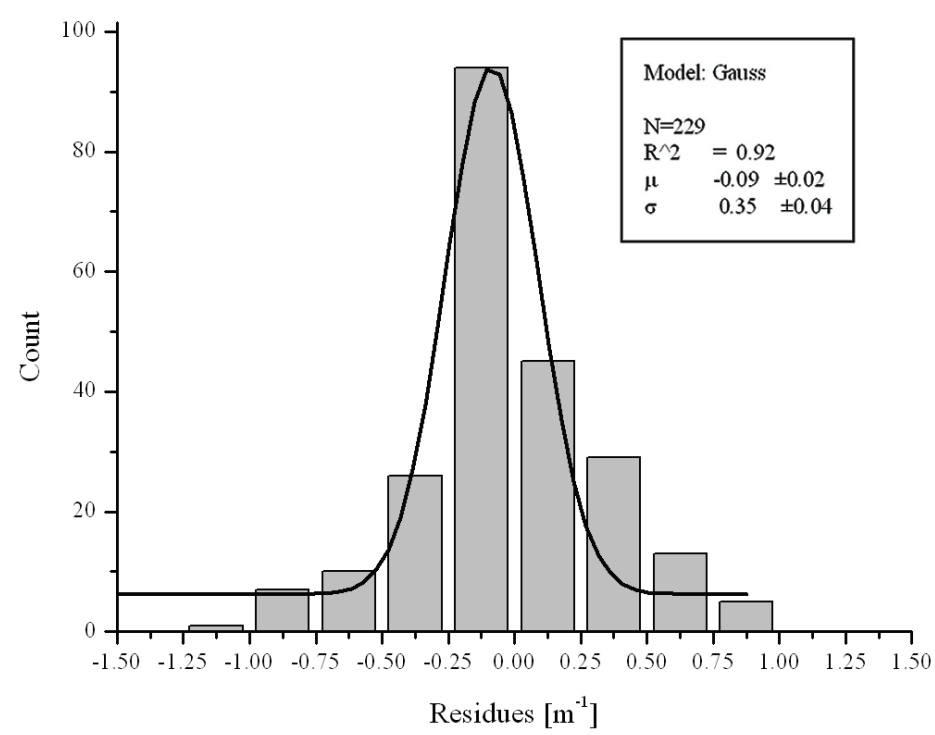

Fig. 4. Distribution of residues between theoretical hyperbolic function and measured related to the PAR diffuse attenuation coefficients and Secchi disk depth data. Solid line represent the best Gaussian distribution.

in equation 10, using the measured $E_{0, P A R}$ just below the surface of the water (Fig. 2). The relation between the 71 values of measured and theoretical $E_{u w, P A R}$ (denoted with $\mathrm{m}$ and $\mathrm{t}$ respectively, Fig. 5), was:

$E_{u w, P A R}^{t}=(0.99 \pm 0.01) \cdot E_{u w, P A R}^{m}$ (Fig. 5) with $R^{2}=0.96$.

This result is found using a value of apparent reflectance $(0.85 \pm 0.04)$ nearest to the actual value of Secchi disk reflectance in the visible band $(\sim 0.80 \pm$ 0.06 ). Considering the differences between the measured reflectance of the Secchi disk and the PAR irradi- ance reflectance we believe that the $0.05 \%$ of difference can be considered in the errors of the estimation. Furthermore, two possible reasons can influence this result in relation to the reflectance of the Secchi disk:

1) in the considered lakes the absorption phenomena is more important than the back scattering phenomena in the extinction of the irradiance (Bracchini et al. 2004, 2005, 2006a, 2006b and 2007)

2) the evidence that the use $K_{d, P A R}$, also called as diffuse attenuation coefficient (e.g., Lee et al. 2005) represent not only the loss of irradiance due to the 


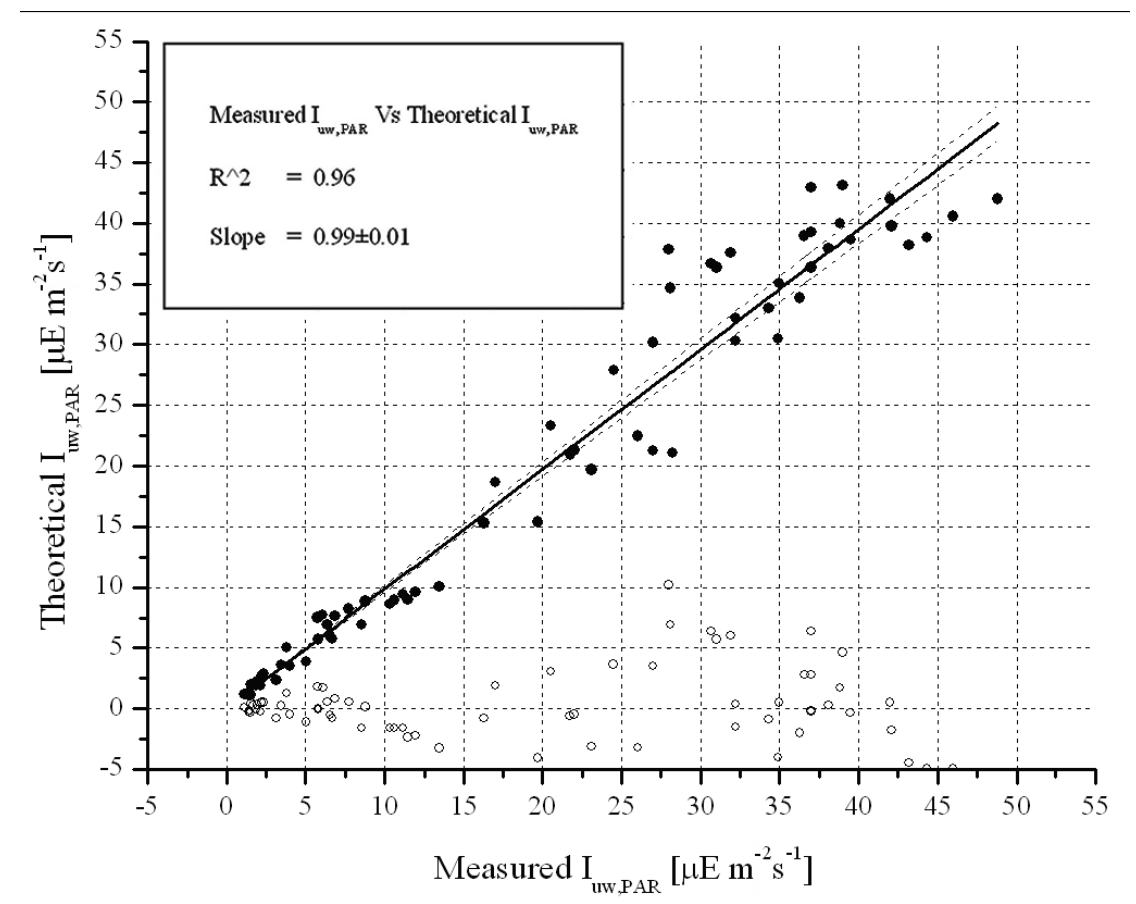

Fig. 5. Measured and theoretical upwelling PAR irradiance (dark circles). Solid line represent the $y=m x$ function $(m \pm \Delta m=0.99 \pm$ 0.01). Dash lines represents the $95 \%$ band confidence. White circles represent the residue values between theoretical and measured upwelling irradiance.

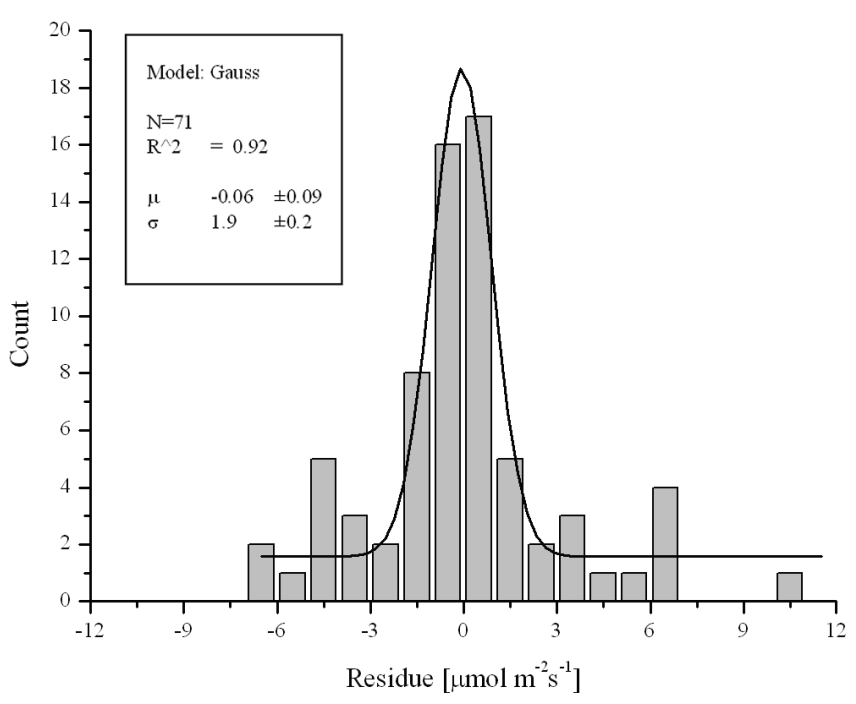

Fig. 6. Gaussian distribution of residue between theoretical and measured upwelling irradiance.

absorption but also the scattering phenomena of the water column between sub surface and the Secchi disk.

Using a one way ANOVA test between the theoretical and measured upwelling irradiances it is possible to conclude that the means are not significantly different with $p \leq 0.81$. The residual distribution between theoretical and measured upwelling irradiances (Fig. 6) reproduces satisfactorily a Gaussian distribution $\left(R^{2}=\right.$ 0.92 , number of data 71 , step size 1.00 ) with expected value $-0.06 \pm 0.09 \mu \mathrm{E} \mathrm{m}^{-2} \mathrm{~s}^{-1}$ and standard deviation 1.9 $\pm 0.2 \mu \mathrm{E} \mathrm{m}^{-2} \mathrm{~s}^{-1}$.

\section{CONCLUSIONS}

The well known relation between Secchi disk depth and visible band attenuation coefficients is confirmed in this work. Despite the different environments and measurements conditions, the value of the CONST = $K_{d, P A R} \times Z_{d}$ is comparable to the values found by others (Raymont 1967; Hojerslev 1986; Edler 1997; Kratzer et 
al. 2003). This suggests a physical and quantitative interpretation of CONST. On the other hand the value of CONST found in this work was different from the value proposed by Padial \& Thomaz (2008) for neotropical ecosystems. The differences between the results could be related by the evidence that no zenithal angle corrections were used in the work of Padial \& Thomaz (2008) but also that the CONST could be dependant by the used data set. Thus, if we choose to represent $K_{d, P A R} \times Z_{d}$ as a constant, we need to introduce an apparent reflectance to take account of the site specificities. Here, we show that the apparent reflectance and the measured reflectance are undistinguishable (if we consider the errors). This result it is probably specific of the used dataset in which rich CDOM water concentrations lakes are used.

The original result of this work is the physicalmathematical interpretation of CONST in terms of the ratio of natural logarithm of upwelling and downwelling irradiance divided by the reflectance (or apparent reflectance) of Secchi disk. Thus, the physical mathematical expression of the CONST allows the evaluation of the PAR upwelling irradiance. The upwelling spectral and band $(10 \mathrm{~nm})$ irradiances are used to estimate phytoplankton productivity, tripton concentration (total inorganic solid) and quality of natural waters (Christian $\&$ Sheng 2003), i.e., in a remote sensing techniques and the estimation of the PAR irradiance using old Secchi disk depth measurements may be useful to retrieve the past temporal dynamics of particles that interact with PAR irradiance (including chlorophyll- $a$ ). The proposed simple model represents the relationship between Secchi disk depth and attenuation coefficient expressed in terms of an hyperbolic function and losing part of absorption and scattering. The debate on the use of a linear (a perfect hyperbolic function), a non linear relationships and with the beam attenuation coefficient (e.g., $\mathrm{K}_{\mathrm{d}, \mathrm{PAR}} \times \mathrm{Z}_{\mathrm{d}}^{\gamma}=$ CONST, with $\gamma \neq 1$ or $c+\mathrm{K}_{\mathrm{d}}=$ $\left.8.69 \times Z_{d}^{-1}\right)$ between Secchi disk depth and attenuation coefficients is still open (Montes-Hugo \& Alvarez-Borrego 2003, 2005; Padial \& Thomaz 2008), but from our results and model interpretation the linear approach appears to be useful to estimate PAR upwelling irradiance.

An high correlation between the measured values of irradiances just below and above water surface is highly probable, so that the result obtained in this work could be used, i.e., in conjunction with remote sense data, to estimate the upwelling irradiance above the water surface knowing the downward irradiance above the water line of ecosystems.

\section{ACKNOWLEDGMENTS}

The data were collected with the financial supporting of EU in a five Scientific Programmes between 2001 and 2004. The authors appreciate the assistance of Dr. Laura Martini for the historical research in relation to the Secchi disk.

\section{REFERENCES}

Bracchini, L., A.M. Dattilo, M. Falcucci, C. Arena \& C. Rossi. 2005. Spatial and temporal variations of the inherent and apparent optical properties in a shallow coastal lake. Journal of Photochemistry and Photobiology B: Biology, 80: 161-177.

Bracchini, L., S.A. Loiselle, A. M. Dattilo, S. Mazzuoli, A. Cózar \& C. Rossi. 2004. The spatial distribution of the optical properties in the UV and Visible in an aquatic ecosystem. Photochemistry and Photobiology, 80: 139-149.

Bracchini, L., A.M. Dattilo, S. Martini,C. Rossi, C. Santinelli \& A. Seritti. 2006b. The bio-optical properties of CDOM as descriptor of lake stratification. Journal of Photochemistry and Photobiology B: Biology, 85: 145-149.

Bracchini, L., A. Cozar, A.M. Dattilo, S.A. Loiselle, A. Tognazzi, N. Azza \& C. Rossi. 2006a. The role of wetlands in the chromophoric dissolved organic matter release and its relation to aquatic ecosystems optical properties. A case of study: Katonga and Bunjako Bays (Victoria Lake; Uganda). Chemosphere, 63: 1170-1178.

Bracchini, L., S.A. Loiselle, A. Tognazzi, A.M. Dattilo, S. Focardi, A. Cozar \& C. Rossi. 2007. The optical qualities of shallow wetland lined bays in Lake Victoria. Wetlands Ecology and Management, 15: 509-519.

Christian, D.Y. \& P. Sheng. 2003. Relative influences of various water quality parameters on light attenuation in Indian lagoon. Estuarine Costal and Shelf Science. 57: 961-971.

Davies-Colley, R., J.W.N. Vant \& D.G. Smith. 1993. Colour and clarity of natural waters: science and management of optical water quality. Ellis Horwood, New York.

Edler, L. 1997. In: Report of the ICES/HELCOM Workshop on quality assurance of pelagic biological measurements in the Baltic Sea. ICES CM 1997/E, 5.

Effler, S.W. 1988. Secchi disc transparency and turbidity. Journal of Environmental Engineering, 114: 1436-1447.

Gordon, H.R.A. \& W. Wouters. 1978. Some relations between Secchi depth and inherent optical properties of natural waters. Applied Optics, 17: 3341-3343.

Haltrin, V.H., D.R.V. Johnson, \& A. Urdenko. 2002. Connection between light field parameters and optical properties of seawater. Ocean Optics: In: G.D. Gilbert \& R.J. Frouin (Eds), Remote Sensing and Underwater Imaging. Volume 4488 .

Holmes, R.W. 1970. The Secchi disk in turbid coastal zones. Limnol. Oceanogr., 15: 688-694.

Hojerslev, N.K. 1986. Visibility of the sea with special reference to the Secchi disc. Proc. Soc. Photo-opt. Instrum. Eng. 637: 294-305.

Hojerslev, N.K. \& T. Aarup. 2002. Optical measurements on the Louisiana Shelf off the Mississippi River. Estuarine, Costal and Shelf Science, 55: 599-611.

Jassby, A.D.C., R. Goldman, J.E. Reuter \& R.C. Richards. 1999. Origins and scale dependence of temporal variability in the transparency of Lake Tahoe, California-Nevada. Limnol. Oceanogr., 44: 282-294.

Kirk, J.T.O. 1984. Dependence of relationship between inherent and apparent optical properties of water on solar altitude. Limnol. Oceanogr., 29: 350-356.

Kirk, J.T.O. 1994. Light \& photosynthesis in aquatic ecosystems. Second Edition. Cambridge University Press.

Lee, Z.P., M. Darecki, K.L. Carder, C.O. Davis, D. Stramski \& W.J. Rhea. 2005. Diffuse attenuation coefficient of downwelling irradiance: An evaluation of remote sensing methods, Journal of Geophysics. Research, 110, C02017, doi: $10.1029 / 2004 J C 002573$.

Mobley, C.D. 1994. Light and water: radiative transfer in natural waters. New York, Academic Press Inc.

Montes-Hugo, M.A., S. Alvarez-Borrego \& A.D. GilesGuzman. 2003. Horizontal sighting and Secchi depth as 
estimator of underwater PAR attenuation in a coastal lagoon. Estuaries, 26: 1302-1309.

Montes-Hugo, M.A.,\& S. Alvarez-Borrego. 2005. Empirical relations to estimate PAR attenuation in San Quintin Bay, using Secchi depth and sighting range. Ciencia Marina, 31: 685-695.

Padial, A.A. \& S.M. Thomaz. 2008. Prediction of the light attenuation coefficient through the Secchi disk depth: empirical modelling in two large Neotropical ecosystems. Limnology, DOI 10.2007/s10201-008-0246-4.

Poole, H.H. \& W.R.G. Atkins. 1929. Photo-electric measurements of submarine illumination throughout the year. $J$. Mar. Biol. Assoc. (UK), 16: 297-324.

Preisendorfer, R. W. 1961. Application of radiative transfer theory to light measurements in the sea. International Union of Geodesy and Geophysics Monographs.

Received: July 2008

Accepted: October 2008
Preisendorfer, R.W. 1986. Secchi disc science: visual optics of natural waters. Limnol. Oceanogr., 31: 909-926.

Sokoletsky, L. 2005. Comparative analysis of selected raditive transfer approaches for aquatic environments. Applied Optics, 44: 136-148.

Tricomi, G.F. 1962. Matematici italiani del primo secolo dello stato unitario. Memorie dell'Accademia delle Scienze di Torino. Classe di Scienze Fisiche, Matematiche e Naturali, Serie IV, Tomo 1.

Tyler, J.E. 1968. The Secchi disc. Limnol. Oceanogr., 13: 1-6. Verschuur, G.L. 1997. Transparency measurements in Garner Lake, Tennessee: The relationship between Secchi depth and solar altitude and a suggestion for normalization of Secchi depth data. Lake and Reservoir Management, 13: 142-153.

Wetzler R.G. \& G.E. Likens. 1991. Limnological analyses. Second edition. Springer-Verlag, New York. 